\title{
Places of misery: mapping slavery of the Dutch East India Company (VOC), 1602-1799
}

\author{
Jeroen Bos ${ }^{\mathrm{a}, *}$ \\ ${ }^{a}$ Groningen University, jeroen.bos1978@gmail.com \\ * Corresponding author
}

Keywords: slavery, allegory, stereotyping, colonial mapping, Dutch East India Company

\begin{abstract}
:
Slavery under the Dutch in the so-called East Indies has - until very recently - been a marginalized topic in the historiography of the long-distance trading company: the Dutch East India Company, or VOC (abbreviated from Verenigde Oost-Indische Compagnie).

Due to several circumstances the study of the Asian perspective in Dutch global slavery has picked up momentum, leading to a boost in scientific and popular literature (among others: Van Rossum 2015). The dedicated exhibition Slavernij (Slavery) in the national history museum Rijksmuseum (May-August 2021) further stimulated public interest, and raised awareness for the topic of Dutch involvement in slavery and slave trade, not excluding the Asian perspective. Several years of research went into preparing the exhibition, also leading to carefully curated publications in Dutch and English (Sint Nicolaas 2021). Another huge incentive for research were the commissions by the authorities of some large Dutch cities who wished thorough investigation into their city's role and involvement in Dutch global slavery (Brandon 2020 , Van Stipriaan 2020, Jouwe 2021). Finally, scientific and public interest for the topic is fuelled by the global Black Lives Matter movement, which also took root in The Netherlands.
\end{abstract}

However, this interest has not yet led to the serious study of maps, plans and topographical views for traces and evidences of slavery and slave trade in the Dutch settlements and occupied territories under the VOC. Other than using them for illustrations to visualise slavery and slave trade in several publications, the sources have barely been used as base for research. This contribution wants to serve as starting point by offering a database (see appendix) in which maps, plans and topographical views are mentioned on which slavery, slave trade, enslaved people (both visualised in literal or allegorical sense), or infrastructure connected to slavery can be observed. The database is intended as helpful tool for all those researching VOC settlements in the broadest sense, and slavery in particular.

The data to fill this database came from the seven volume spanning series Comprehensive atlas of the United Dutch East India Company (Van Diessen 2006-2010). It is a facsimile publication of all known manuscript maps, plans, and topographical views of the Dutch overseas settlements. The data is presented by volume/page number, inventory number of the work, geographical region, city or town, year of production, mapmaker(s) (if known), type (infrastructure or depiction), followed by the original description that can be found on the work. The Comprehensive atlas will be made available in open access by the National Archives of the Netherlands in the near future.

Four small case studies will be presented as examples of how maps, plans and topographical views can be used as base for research The first will handle the depiction of infrastructure in the Dutch mining facilities at the island of Sumatra (Silida). The second case study will dive into the topic of gender, focussing on references of housing for enslaved women (the Dutch word for an enslaved woman is 'slavin') and looking for reasons why mapmakers mentioned this specifically. The third case study is about stereotyping. The work of artist Johannes Rach (1720-1783) was popular among contemporaries. A set of city views of Batavia will be discussed for the depiction of enslaved people, with special attention to followers and copyists of Rach. Unlike Rach, who travelled the Dutch empire in Asia, these copyists worked in the Dutch Republic and stereotyped enslaved people, even adding elements (like a typical black servant boy wearing orientalised dress) not present on the original drawings by Rach. In the fourth case study "remoteness" will be examined by looking at smaller Dutch settlements in Asia (Vengurla, Bimilipatnam), refuting traditional notions that Dutch slavery in Asia was to be found exclusively at the metropoles.

Finally, for the advancement of the study of slavery in general it is important to collect contemporaries' views and opinions on the topic. Remarkably enough the testimony of a VOC mapmaker has survived the ages in the archival records of the Company at the National Archives of the Netherlands (NA). The Prussian-born Carl Friedrich Reimer (c. 
1740-1796) became the most productive in-situ mapmaker for the VOC (Bos 2020). In his records can be found his personal opinion about slavery on the island of Java. It offers a unique view on the matter, laced with late $18^{\text {th }}$ century Enlightenment ideas. Testimonies like the one by Reimer are rare, and found mainly by serendipity. The ongoing investment from the NA in artificial intelligence in handwritten text recognition (HTR) software means that the VOC handwritten records can be made searchable for keywords. This is important to reconstruct the link between many VOC maps and the original accompanying reports in the collections of the NA. In the $19^{\text {th }}$ century archivist P.A. Leupe decided to separate maps and plans of the former VOC and WIC from the original records and store them separately. A decision that made sense in the late $19^{\text {th }}$ century, but regretted nowadays. Only with painstakingly archival research the broken link between map and report can be reconstructed. It is expected that HTR will ease this reconstruction. Likewise, anticipation is high that artificial intelligence will reveal many hidden stories, like Reimers testimony on slavery, from obscurity. A very promising future indeed.

\section{Acknowledgements}

The author likes to thank curator of maps and atlases Martijn Storms (Leiden University Libraries) for initial talks and thoughts about this topic.

\section{References}

Bos, J., 2020. 'Dead on Arrival': The Unused Cartographic Legacy of Carl Friedrich Reimer. In: Kent A., Vervust S., Demhardt I., Millea N. (eds) Mapping Empires: Colonial Cartographies of Land and Sea. Lecture Notes in Geoinformation and Cartography. Springer, Cham, pp. 287-307. https://doi.org/10.1007/978-3-030-23447-8_16

Brandon, P. (ed), 2020. De slavernij in Oost en West: het Amsterdam onderzoek. Spectrum, Amsterdam.

Jouwe, N. (ed), 2021. Slavernij en de stad Utrecht. Walburg, Zutphen.

Van Diessen, R. (ed), 2006-2010. Comprehensive atlas of the United Dutch East India Company. Asia Maior/Atlas Maior, Voorburg.

Van Rossum, M., 2015. Kleurrijke tragiek. De geschiedenis van slavernij in Azië onder de VOC. Verloren, Hilversum. Van Stipriaan, A., 2020. Rotterdam in slavernij. Boom, Amsterdam.

\section{Appendix}

Database slavery on manuscript VOC maps, plans and views. 


\section{Appendix 1}

\begin{tabular}{|c|c|c|c|c|c|c|c|}
\hline $\begin{array}{l}\text { SERIES } \\
\text { VOLUME }\end{array}$ & $\begin{array}{l}\text { INSTITUTIONAL } \\
\text { INV NR }\end{array}$ & REGION & CITY/TOWN/LOCALITY & YEAR(S) & MAPMAKER(S) & TYPE & $\begin{array}{l}\text { ORIGINAL } \\
\text { DESCRIPTION }\end{array}$ \\
\hline I I-5c & NA 4.VEL-828 & Africa & Cape of Good Hope & c. 1700 & Isaak de Graaf & infrastructure & $\begin{array}{l}\text { Slavenhuysen; 't } \\
\text { ontwerp van een } \\
\text { slavenhuys } \\
\text { welkers } \\
\text { fundament } \\
\text { muuren mede } \\
\text { bijna geleyt sijn; } \\
\text { Loots voor de } \\
\text { slaven }\end{array}$ \\
\hline I I-5d & NA 4.VEL-830 & Africa & Cape of Good Hope & c. 1700 & Isaak de Graaf & infrastructure & $\begin{array}{l}\text { Een provisioneel } \\
\text { slavenhuys } \\
\text { konnende na } \\
\text { desen tot smiths, } \\
\text { wagenmakers, } \\
\text { en kuypers } \\
\text { winkels } \\
\text { gebruykt werden }\end{array}$ \\
\hline I I-17 & NA 4.VEL-885 & India & Vengurla & c. 1700 & Isaak de Graaf & infrastructure & $\begin{array}{l}\text { eenige } \\
\text { wooninge } \\
\text { binnen 't begrip } \\
\text { van de logie zo } \\
\text { voor de } \\
\text { thuyniers, } \\
\text { slaven, etc. }\end{array}$ \\
\hline I I-23g & NA 4.VEL-986 & $\begin{array}{l}\text { Sri } \\
\text { Lanka }\end{array}$ & $\begin{array}{l}\text { Colombo (Governor's } \\
\text { House) }\end{array}$ & c. 1700 & Isaak de Graaf & infrastructure & $\begin{array}{l}\text { Een afdak tot } \\
\text { logiment der } \\
\text { lijfeygenen; vier } \\
\text { secreeten en } \\
\text { washuysjiens } \\
\text { voor de slaven; } \\
\text { een trap langs } \\
\text { welke de slaven } \\
\text { en het vee na } \\
\text { boven tot de } \\
\text { gemeene grondt } \\
\text { opgaan }\end{array}$ \\
\hline I I-25c & NA 4.VEL-1083 & India & Nagapatnam & c. 1700 & Isaak de Graaf & infrastructure & $\begin{array}{l}\text { Slavekamerties; } \\
\text { slave kamer; der } \\
\text { slaven secreet; } \\
\text { slavenkamer; } \\
\text { slave kamer; } \\
\text { slave secreet; } \\
\text { slavensecreet }\end{array}$ \\
\hline I I-25e & NA 4.VEL-1087 & India & Sadrangapatnam & c. 1700 & Isaak de Graaf & infrastructure & $\begin{array}{l}\text { Combuysen en } \\
\text { slavenkotten }\end{array}$ \\
\hline I I-25e & NA 4.VEL-1087 & India & Porto Novo & c. 1700 & Isaak de Graaf & infrastructure & $\begin{array}{l}\text { Combuysen en } \\
\text { slavenkotten }\end{array}$ \\
\hline I I-29b & NA 4.VEL-1101 & India & Houghly & c. 1700 & Isaak de Graaf & infrastructure & $\begin{array}{l}\text { Combuysen en } \\
\text { kuffen voor de } \\
\text { slaven }\end{array}$ \\
\hline I I-36b & NA 4.VEL-1145 & Indonesia & Cinkuk Gadang & c. 1700 & Isaak de Graaf & infrastructure & $\begin{array}{l}\text { T woonhuys } \\
\text { voor s' Comp:s } \\
\text { slaven, } \\
\text { slavinnen en } \\
\text { banditen }\end{array}$ \\
\hline I I-36b & NA 4.VEL-1145 & Indonesia & Barus & c. 1700 & Isaak de Graaf & infrastructure & $\begin{array}{l}\text { Der slaven } \\
\text { wooningh }\end{array}$ \\
\hline I II-24 & NA 4.VEL-1341 & Indonesia & Saparua & c. 1700 & Isaak de Graaf & infrastructure & Slavenhuys \\
\hline I II-26a & NA 4.VEL-1359 & Indonesia & Bandanaira & c. 1700 & Isaak de Graaf & infrastructure & Slaven quartier \\
\hline II 28-29 & $\begin{array}{l}\text { UBL Bodel } \\
\text { Nijenhuis Port } 56 \\
\text { N } 59\end{array}$ & Indonesia & Java & 1718 & $\begin{array}{l}\text { Hadriano } \\
\text { Relando }\end{array}$ & allegorical & $\begin{array}{l}\text { [depiction of } \\
\text { enslaved people } \\
\text { in the cartouche, } \\
\text { allegorical] }\end{array}$ \\
\hline
\end{tabular}




\begin{tabular}{|c|c|c|c|c|c|c|c|}
\hline II 96 & $\begin{array}{l}\text { Westfries Museum } \\
02020\end{array}$ & Indonesia & Batavia & c. 1627 & Anonymous & infrastructure & $\begin{array}{l}\text { Huysing van des } \\
\text { Comp [slaven] }\end{array}$ \\
\hline II 99 & $\begin{array}{l}\text { Atlas van Stolk, } \\
\text { No. } 1672\end{array}$ & Indonesia & Batavia & 1629 & Gilles Venant & infrastructure & $\begin{array}{l}\text { T Nieuwe } \\
\text { quartier van } \\
\text { slaven }\end{array}$ \\
\hline II 122 & NA 4.VEL 1232 & Indonesia & Batavia (Castle) & c. 1698 & anonymous & infrastructure & $\begin{array}{l}\text { Sekreet voor de } \\
\text { slaven }\end{array}$ \\
\hline II 123-a & $\begin{array}{l}\text { Rijksmuseum, SK- } \\
\text { A-1901 }\end{array}$ & Indonesia & Batavia & 1663 & $\begin{array}{l}\text { Andries } \\
\text { Beeckman }\end{array}$ & depiction & $\begin{array}{l}\text { [depiction of } \\
\text { enslaved people } \\
\text { on the market of } \\
\text { Batavia] }\end{array}$ \\
\hline II 123-b & KIT, 118-167 & Indonesia & Batavia & c. 1656 & $\begin{array}{l}\text { Andries } \\
\text { Beeckman } \\
\text { (attributed) }\end{array}$ & depiction & $\begin{array}{l}\text { [depiction of } \\
\text { enslaved people } \\
\text { on the market of } \\
\text { Batavia] }\end{array}$ \\
\hline II $126-b$ & $\begin{array}{l}\text { Atlas van Stolk, } \\
\text { TOP } 1043\end{array}$ & Indonesia & Batavia & 1779 & Johannes Rach & depiction & $\begin{array}{l}\text { [depiction of } \\
\text { enslaved people, } \\
\text { working] }\end{array}$ \\
\hline II $127-\mathrm{a}$ & $\begin{array}{l}\text { Rijksmuseum, RP- } \\
\text { T-FM-74 }\end{array}$ & indonesia & Batavia & $1775-1779$ & $\begin{array}{l}\text { Hendrik Kobell } \\
\text { jr }\end{array}$ & depiction & $\begin{array}{l}\text { [depiction of } \\
\text { enslaved people, } \\
\text { working] }\end{array}$ \\
\hline II $128-\mathrm{a}$ & PNRI, BW 3 & Indonesia & Batavia & $1772-1775$ & Johannes Rach & depiction & $\begin{array}{l}\text { [depiction of } \\
\text { enslaved people, } \\
\text { working] }\end{array}$ \\
\hline II 129-a & $\begin{array}{l}\text { Rijksmuseum, RP- } \\
\text { T-FM-75 }\end{array}$ & Indonesia & Batavia & $1775-1779$ & $\begin{array}{l}\text { Hrendrik Kobell } \\
\text { jr }\end{array}$ & depiction & $\begin{array}{l}\text { [depiction of } \\
\text { enslaved people, } \\
\text { working] }\end{array}$ \\
\hline II 172-a & $\begin{array}{l}\text { Atlas van Stolk, } \\
\text { TOP } 1045\end{array}$ & Indonesia & Batavia & 1764 & Johannes Rach & depiction & $\begin{array}{l}\text { [depiction of } \\
\text { enslaved people, } \\
\text { working] }\end{array}$ \\
\hline II 187-b & ANRI, H 82 & Indonesia & Batavia (Onrust) & 1803 & $\begin{array}{l}\text { Dominicus } \\
\text { Michel Barbier }\end{array}$ & infrastructure & $\begin{array}{l}\text { Logie der } \\
\text { lijfeigene }\end{array}$ \\
\hline II 192-a & NA 4.VMF 849.7 & Indonesia & Edam Island & c. 1780 & Anonymous & infrastructure & $\begin{array}{l}\text { Gevangenis } \\
\text { voor Slavinen; } \\
\text { Banditen huijs }\end{array}$ \\
\hline II 246 & NA 4.VEL 1171 & Indonesia & Karawang & 1730 & Anonymous & infrastructure & Slaven Quartier \\
\hline II 262-b & PNRI, BWN 6 & Indonesia & Batavia (Molenvliet) & ca. 1778 & Johannes Rach & depiction & $\begin{array}{l}\text { [depiction of } \\
\text { enslaved people, } \\
\text { working] }\end{array}$ \\
\hline II 366 & NA 4.VEL 1264 & Indonesia & Semarang & 1787 & Anonymous & infrastructure & $\begin{array}{l}\text { Gevangen huijs } \\
\text { voor de } \\
\text { gecomdenneerde } \\
\text { Slaven }\end{array}$ \\
\hline II 383-b & NA 4.VEL 1276 & Indonesia & Rembang & c. 1709 & Anonymous & infrastructure & $\begin{array}{l}\text { Woonhuijs } \\
\text { Gemaakt voor } \\
\text { de inlanders }\end{array}$ \\
\hline III 57-b & NA 4.VEL 1144 & Indonesia & Silada & 1737 & $\begin{array}{l}\text { C. Stiegehuis, F. } \\
\text { Markscheider }\end{array}$ & infrastructure & $\begin{array}{l}\text { Quartieren voor } \\
\text { 's Comp: slaven }\end{array}$ \\
\hline $\begin{array}{l}\text { III } 114- \\
115\end{array}$ & NA 4.VEL 1113 & Indonesia & Malacca & 1791 & $\begin{array}{l}\text { P. Elias (copy of } \\
\text { C.F. Reimer) }\end{array}$ & infrastructure & $\begin{array}{l}\text { S Komps } \\
\text { sclaven } \\
\text { Vertrecken }\end{array}$ \\
\hline III 136 & NA 4.MIKO 164 & Indonesia & Banjarmasin & $1810-1830$ & $\begin{array}{l}\text { Jan Theunis } \\
\text { Busscher }\end{array}$ & infrastructure & Slaaven Vertrek \\
\hline $\begin{array}{l}\text { III } 162- \\
163\end{array}$ & NA 4.VEL 1293 & Indonesia & Macassar & 1693 & Anonymous & depiction & $\begin{array}{l}\text { [depiction of } \\
\text { enslaved people } \\
\text { in the cartouche, } \\
\text { allegorical] }\end{array}$ \\
\hline III 189 & NA 4.VEL 1304 & Indonesia & Bantimurung & 1735 & F.J. Steinfeldt & infrastructure & $\begin{array}{l}\text { Huijsen voor de } \\
\text { Bergluiden }\end{array}$ \\
\hline
\end{tabular}




\begin{tabular}{|c|c|c|c|c|c|c|c|}
\hline $\begin{array}{l}\text { III } 228- \\
\text { a/b }\end{array}$ & $\begin{array}{l}\text { KIT, 3728-540A / } \\
\text { KIT, 3728-541A }\end{array}$ & Indonesia & Kupang (Fort Concordia) & 1756 & Anonymous & depiction & $\begin{array}{l}\text { depiction of } \\
\text { enslaved people, } \\
\text { working] }\end{array}$ \\
\hline III 251 & NA 4.VELH 555 & Indonesia & Ternate (Castle Orange) & c. 1663 & Anonymous & infrastructure & Slavenhuijs \\
\hline IV $42-43$ & NA 4.MIKO W42 & $\begin{array}{l}\text { Sri } \\
\text { Lanka }\end{array}$ & Ceylon (whole) & 1751 & $\begin{array}{l}\text { Baltus Jacobsz } \\
\text { van Lier }\end{array}$ & depiction & $\begin{array}{l}\text { [cinnamom } \\
\text { carriers and } \\
\text { slaves (?) in } \\
\text { cartouche, } \\
\text { allegorical] }\end{array}$ \\
\hline IV $60 \mathrm{~b}$ & $\begin{array}{l}\text { Centrale } \\
\text { Bibliotheek } \\
\text { Rotterdam, 86 L } 8\end{array}$ & $\begin{array}{l}\text { Sri } \\
\text { Lanka }\end{array}$ & Colombo & 1688 & $\begin{array}{l}\text { Jan Christiaensz } \\
\text { Toorzee }\end{array}$ & infrastructure & $\begin{array}{l}\text { School van s' } \\
\text { Comp:s } \\
\text { lijffeijgenen; } \\
\text { Slaven } \\
\text { hospitaal; } \\
\text { Slaven quartier }\end{array}$ \\
\hline IV 63 & NA 4.VEL 945B & $\begin{array}{l}\text { Sri } \\
\text { Lanka }\end{array}$ & Colombo & 1693 & $\begin{array}{l}\text { Jan Christiaensz } \\
\text { Toorzee }\end{array}$ & infrastructure & $\begin{array}{l}\text { [in the } \\
\text { description:] d' } \\
\text { Sluijs QR, en } \\
\text { het daar neven } \\
\text { uitgaande } \\
\text { poortie nae 't } \\
\text { Slaven quartier } \\
\text { AP }\end{array}$ \\
\hline $\begin{array}{l}\text { IV 64-65- } \\
\text { a }\end{array}$ & NA 4.VEL 945 & $\begin{array}{l}\text { Sri } \\
\text { Lanka }\end{array}$ & Colombo & 1697 & $\begin{array}{l}\text { Jan Christiaensz } \\
\text { Toorzee }\end{array}$ & infrastructure & $\begin{array}{l}\text { Brug om na 't } \\
\text { Slaven quartier } \\
\text { te gaan; Slaven } \\
\text { Quartier }\end{array}$ \\
\hline $\begin{array}{l}\text { IV 64-65- } \\
\text { b }\end{array}$ & NA 4.VEL 946 & $\begin{array}{l}\text { Sri } \\
\text { Lanka }\end{array}$ & Colombo & 1697 & $\begin{array}{l}\text { Jan Christiaensz } \\
\text { Toorzee }\end{array}$ & infrastructure & $\begin{array}{l}\text { Bruggen na 't } \\
\text { Slaven Quartier; } \\
\text { Slaven Quartier }\end{array}$ \\
\hline IV 66 & NA 4.VEL 947A & $\begin{array}{l}\text { Sri } \\
\text { Lanka }\end{array}$ & Colombo & 1697 & $\begin{array}{l}\text { Jan Christiaensz } \\
\text { Toorzee }\end{array}$ & infrastructure & $\begin{array}{l}\text { Poortie om na 't } \\
\text { Slaven Eijland } \\
\text { te gaan; } \\
\text { Bruggetje om na } \\
\text { 't Slaven Eijland } \\
\text { te gaan }\end{array}$ \\
\hline IV 69 & NA 4.VEL 948 & $\begin{array}{l}\text { Sri } \\
\text { Lanka }\end{array}$ & Colombo & 1698 & $\begin{array}{l}\text { Jan Christiaensz } \\
\text { Toorzee }\end{array}$ & infrastructure & $\begin{array}{l}\text { Poortje om na 't } \\
\text { Slaven Eijland } \\
\text { te gaan; } \\
\text { Bruggetje om na } \\
\text { 't Slaven Eijland } \\
\text { te gaan }\end{array}$ \\
\hline IV 71-a & $\begin{array}{l}\text { UBL, Bodel } \\
\text { Nijenhuis 002-11- } \\
065\end{array}$ & $\begin{array}{l}\text { Sri } \\
\text { Lanka }\end{array}$ & Colombo & c. 1721 & Anonymous & infrastructure & $\begin{array}{l}\text { Co[mpagnies] } \\
\text { Slaven Neg[erij] }\end{array}$ \\
\hline IV 74 & NA 4.MIKO W24 & $\begin{array}{l}\text { Sri } \\
\text { Lanka }\end{array}$ & Colombo & 1753 & $\begin{array}{l}\text { Baltus Jacobsz } \\
\text { van Lier }\end{array}$ & infrastructure & $\begin{array}{l}\text { [on map:] } \\
\text { Slaven quartier }\end{array}$ \\
\hline IV 75 & NA 4.MIKO W25 & $\begin{array}{l}\text { Sri } \\
\text { Lanka }\end{array}$ & Colombo & 1756 & $\begin{array}{l}\text { Baltus Jacobsz } \\
\text { van Lier }\end{array}$ & infrastructure & $\begin{array}{l}\text { Het 's Comp:s } \\
\text { Slaven quartier }\end{array}$ \\
\hline IV 77 & NA 4.VELH 335 & $\begin{array}{l}\text { Sri } \\
\text { Lanka }\end{array}$ & Colombo & $1760-1765$ & $\begin{array}{l}\text { Christiaan } \\
\text { Conrad } \\
\text { Wohlfarth }\end{array}$ & infrastructure & $\begin{array}{l}\text { s E: Comps. } \\
\text { [Slaven] } \\
\text { Quartier }\end{array}$ \\
\hline IV 78-79 & NA 4.VEL 953 & $\begin{array}{l}\text { Sri } \\
\text { Lanka }\end{array}$ & Colombo & $1762-1765$ & $\begin{array}{l}\text { Carel David } \\
\text { Wentzel }\end{array}$ & infrastructure & $\begin{array}{l}\text { s E: Comps. } \\
\text { Slaaven Quartier }\end{array}$ \\
\hline IV 80 & NA 4.VEL 954 & $\begin{array}{l}\text { Sri } \\
\text { Lanka }\end{array}$ & Colombo & 1785 & $\begin{array}{l}\text { Samuel Pieter } \\
\text { Foenander }\end{array}$ & infrastructure & $\begin{array}{l}\text { [on map:] } \\
\text { Slaven quartier }\end{array}$ \\
\hline IV 86 & NA 4.VEL 962 & $\begin{array}{l}\text { Sri } \\
\text { Lanka }\end{array}$ & Colombo & 1787 & $\begin{array}{l}\text { Elias Paravicini } \\
\text { di Capelli }\end{array}$ & infrastructure & $\begin{array}{l}\text { [in the } \\
\text { description:] De } \\
\text { Afsnijdingen } \\
\text { van een gedeelte } \\
\text { van den } \\
\text { Voorgrond aan } \\
\text { den Oost en } \\
\text { West Zijde so } \\
\text { wel als van het } \\
\text { Slaven } \\
\text { Eijland... }\end{array}$ \\
\hline
\end{tabular}




\begin{tabular}{|c|c|c|c|c|c|c|c|}
\hline IV $86-87$ & NA 4.VEL 964A & $\begin{array}{l}\text { Sri } \\
\text { Lanka }\end{array}$ & Colombo & 1787 & De La Lustrière & infrastructure & $\begin{array}{l}\text { Quartier des } \\
\text { ésclaves de la } \\
\text { Compagnie }\end{array}$ \\
\hline IV 89 & NA 4.VEL 968A & $\begin{array}{l}\text { Sri } \\
\text { Lanka }\end{array}$ & Colombo & 1787 & $\begin{array}{l}\text { Carl Friedrich } \\
\text { Reimer }\end{array}$ & infrastructure & $\begin{array}{l}\text { [on map:] 't } \\
\text { Slaven Eyland }\end{array}$ \\
\hline IV 90 & NA 4.VEL 966 & $\begin{array}{l}\text { Sri } \\
\text { Lanka }\end{array}$ & Colombo & 1787 & $\begin{array}{l}\text { Carl Friedrich } \\
\text { Reimer }\end{array}$ & infrastructure & $\begin{array}{l}\text { [on map:] 't } \\
\text { Slaven Eyland }\end{array}$ \\
\hline IV 99 & NA 4.VEL 978 & $\begin{array}{l}\text { Sri } \\
\text { Lanka }\end{array}$ & Colombo & 1789 & $\begin{array}{l}\text { Carl Friedrich } \\
\text { Reimer }\end{array}$ & infrastructure & $\begin{array}{l}\text { [on map:] Het } \\
\text { Slaven Eyland }\end{array}$ \\
\hline IV 100-b & NA 4.VEL 980A & $\begin{array}{l}\text { Sri } \\
\text { Lanka }\end{array}$ & Colombo & 1790 & $\begin{array}{l}\text { P. Elias (copy of } \\
\text { G.E. Schenk) }\end{array}$ & infrastructure & $\begin{array}{l}\text { [on map:] Het } \\
\text { Slaven Eyland }\end{array}$ \\
\hline IV 101 & NA 4.VEL 975 & $\begin{array}{l}\text { Sri } \\
\text { Lanka }\end{array}$ & Colombo & 1790 & $\begin{array}{l}\text { G.E. Schenk } \\
\text { (copy of C.F. } \\
\text { Reimer) }\end{array}$ & infrastructure & $\begin{array}{l}\text { [on map:] Het } \\
\text { Slaven Eyland }\end{array}$ \\
\hline IV $112-\mathrm{a}$ & PNRI, BWN 32 & $\begin{array}{l}\text { Sri } \\
\text { Lanka }\end{array}$ & Colombo & $1775-1780$ & Johannes Rach & depiction & $\begin{array}{l}\text { [depiction of } \\
\text { enslaved people, } \\
\text { working] }\end{array}$ \\
\hline IV 112-b & $\begin{array}{l}\text { Rijksmuseum, RP- } \\
\text { T-1904-18 }\end{array}$ & $\begin{array}{l}\text { Sri } \\
\text { Lanka }\end{array}$ & Colombo & 1772 & $\begin{array}{l}\text { Carl Friedrich } \\
\text { Reimer }\end{array}$ & depiction & $\begin{array}{l}\text { [depiction of } \\
\text { enslaved people, } \\
\text { working] }\end{array}$ \\
\hline IV 114-a & KIT, $48 \mathrm{M}-7$ & $\begin{array}{l}\text { Sri } \\
\text { Lanka }\end{array}$ & Colombo (Hospital) & c. 1771 & Anonymous & depiction & $\begin{array}{l}\text { [depiction of } \\
\text { enslaved people, } \\
\text { working] }\end{array}$ \\
\hline IV 119-a & $\begin{array}{l}\text { Rijksmuseum, RP- } \\
\text { T-00-3250 }\end{array}$ & $\begin{array}{l}\text { Sri } \\
\text { Lanka }\end{array}$ & Colombo & c. 1755 & Anonymous & depiction & $\begin{array}{l}\text { [depiction of } \\
\text { enslaved people, } \\
\text { working] }\end{array}$ \\
\hline IV 119-b & $\begin{array}{l}\text { Rijksmuseum, RP- } \\
\text { T-00-3247 }\end{array}$ & $\begin{array}{l}\text { Sri } \\
\text { Lanka }\end{array}$ & Colombo & c. 1755 & Anonymous & depiction & $\begin{array}{l}\text { [depiction of } \\
\text { enslaved people, } \\
\text { working] }\end{array}$ \\
\hline IV $120-b$ & $\begin{array}{l}\text { Rijksmuseum, } \\
\text { NG-1985-7-3-18 }\end{array}$ & $\begin{array}{l}\text { Sri } \\
\text { Lanka }\end{array}$ & Colombo & 1785 & Jan Brandes & depiction & $\begin{array}{l}\text { [depiction of } \\
\text { enslaved people, } \\
\text { working] }\end{array}$ \\
\hline IV $120-b$ & $\begin{array}{l}\text { [private } \\
\text { collection] }\end{array}$ & $\begin{array}{l}\text { Sri } \\
\text { Lanka }\end{array}$ & Colombo & 1785 & Jan Brandes & depiction & $\begin{array}{l}\text { [depiction of } \\
\text { enslaved people, } \\
\text { working] }\end{array}$ \\
\hline IV 141-b & $\begin{array}{l}\text { Rijksmuseum, } \\
\text { NG-1985-7-2-100 }\end{array}$ & $\begin{array}{l}\text { Sri } \\
\text { Lanka }\end{array}$ & Toppuwa & 1785 & Jan Brandes & depiction & $\begin{array}{l}\text { [depiction of } \\
\text { enslaved people, } \\
\text { working] }\end{array}$ \\
\hline IV 143-b & $\begin{array}{l}\text { Rijksmuseum, } \\
\text { NG-1985-7-2- } \\
86 / 87\end{array}$ & $\begin{array}{l}\text { Sri } \\
\text { Lanka }\end{array}$ & Toppuwa & 1785 & Jan Brandes & depiction & $\begin{array}{l}\text { [depiction of } \\
\text { enslaved people, } \\
\text { working] }\end{array}$ \\
\hline IV 166-b & $\begin{array}{l}\text { Rijksmuseum, RP- } \\
\text { T-00-3248 }\end{array}$ & $\begin{array}{l}\text { Sri } \\
\text { Lanka }\end{array}$ & Kalutara & c. 1755 & Anonymous & depiction & $\begin{array}{l}\text { [depiction of } \\
\text { enslaved people, } \\
\text { working] }\end{array}$ \\
\hline $\begin{array}{l}\text { IV } 190- \\
191\end{array}$ & NA 4.VEL 1059 & $\begin{array}{l}\text { Sri } \\
\text { Lanka }\end{array}$ & Galle & 1787 & De La Lustrière & infrastructure & $\begin{array}{l}\text { Hôpital des } \\
\text { esclaves e la } \\
\text { compagnie qui } \\
\text { sert de cazernes } \\
\text { aux troupes } \\
\text { Malaises }\end{array}$ \\
\hline IV 200 & NA 4.VEL 1071 & $\begin{array}{l}\text { Sri } \\
\text { Lanka }\end{array}$ & Galle & 1790 & G.E. Schenk & infrastructure & Slaven hospitaal \\
\hline IV 291-b & $\begin{array}{l}\text { Rijksmuseum, RP- } \\
\text { T-1902-A-4670 }\end{array}$ & $\begin{array}{l}\text { Sri } \\
\text { Lanka }\end{array}$ & Jaffnapatnam & c. 1730 & [Caspar Steiger] & depiction & $\begin{array}{l}\text { [depiction of } \\
\text { enslaved people, } \\
\text { working] }\end{array}$ \\
\hline IV 292-a & $\begin{array}{l}\text { Rijksmuseum, RP- } \\
\text { T-1902-A-4661 }\end{array}$ & $\begin{array}{l}\text { Sri } \\
\text { Lanka }\end{array}$ & Jaffnapatnam & c. 1730 & [Caspar Steiger] & depiction & $\begin{array}{l}\text { [depiction of } \\
\text { enslaved people, } \\
\text { working] }\end{array}$ \\
\hline IV 291-b & $\begin{array}{l}\text { Rijksmuseum, RP- } \\
\text { T-1902-A-4664 }\end{array}$ & $\begin{array}{l}\text { Sri } \\
\text { Lanka }\end{array}$ & Jaffnapatnam & c. 1730 & [Caspar Steiger] & depiction & $\begin{array}{l}\text { [depiction of } \\
\text { enslaved people, } \\
\text { working] }\end{array}$ \\
\hline
\end{tabular}




\begin{tabular}{|c|c|c|c|c|c|c|c|}
\hline IV 294-a & $\begin{array}{l}\text { NA 1.04.02 9714, } \\
\text { fols. } 1422-1423\end{array}$ & $\begin{array}{l}\text { Sri } \\
\text { Lanka }\end{array}$ & Nallur & 1690 & Anonymous & infrastructure & $\begin{array}{l}\text { Slave plaats van } \\
\text { d[itt]o }\end{array}$ \\
\hline IV 324-a & NA 4.MIKO W31 & $\begin{array}{l}\text { Sri } \\
\text { Lanka }\end{array}$ & Trincomalee & 1756 & $\begin{array}{l}\text { Baltus Jacobsz } \\
\text { van Lier }\end{array}$ & infrastructure & $\begin{array}{l}\text { Slaven } \\
\text { hos[pitaal] en } \\
\text { Smitswinkel }\end{array}$ \\
\hline IV 334-a & NA 4.MIKO 29 & $\begin{array}{l}\text { Sri } \\
\text { Lanka }\end{array}$ & Trincomalee & c. 1756 & $\begin{array}{l}\text { Baltus Jacobsz } \\
\text { van Lier } \\
\text { (attributed) }\end{array}$ & infrastructure & $\begin{array}{l}\text { Slaaven } \\
\text { hospitaal en } \\
\text { Smitswinkel }\end{array}$ \\
\hline IV 335 & NA 4.MIKO 28 & $\begin{array}{l}\text { Sri } \\
\text { Lanka }\end{array}$ & Trincomalee & $1755-1760$ & Anonymous & infrastructure & $\begin{array}{l}\text { Het slaven } \\
\text { Hospitaal }\end{array}$ \\
\hline V 75 & NA 4.VEL 824 & Africa & Cape Colony & c. 1660 & Pieter Potter & infrastructure & $\begin{array}{l}\text { Compagnies } \\
\text { gemene volcx } \\
\text { ende slave } \\
\text { Combuijs ende } \\
\text { backerij }\end{array}$ \\
\hline V 78 & NA 4.VEL 826 & Africa & Cape Colony & c. 1663 & $\begin{array}{l}\text { Zacharias } \\
\text { Wagenaer }\end{array}$ & infrastructure & $\begin{array}{l}\text { De keuken } \\
\text { daerin } \\
\text { dagelijcks voor } \\
\text { de siecken en } \\
\text { des E. Comp:s } \\
\text { slaven gekoockt } \\
\text { wort, mitsgaders } \\
\text { 't brouw en } \\
\text { Backhuijs al } \\
\text { onder een } \\
\text { schoorsteen te } \\
\text { brengen }\end{array}$ \\
\hline V 79 & NA 4.VEL 825 & Africa & Cape Colony & c. 1665 & Hendricq Lacus & infrastructure & $\begin{array}{l}\text { Comp:s gemene } \\
\text { volcx en slaven } \\
\text { Combuijs en } \\
\text { Backerij }\end{array}$ \\
\hline V 95-b & PNRI, BWN 27-A & Africa & Cape Colony & $1762-1780$ & $\begin{array}{l}\text { Johannes Rach, } \\
\text { apprentice or } \\
\text { follower }\end{array}$ & depiction & $\begin{array}{l}\text { [depiction of } \\
\text { enslaved people, } \\
\text { working] }\end{array}$ \\
\hline $\begin{array}{l}\text { V 100- } \\
101\end{array}$ & $\begin{array}{l}\text { UBL, Bodel } \\
\text { Nijenhuis 006-25- } \\
031 / 38\end{array}$ & Africa & Cape Colony & c. 1760 & $\begin{array}{l}\text { Carel David } \\
\text { Wentzel } \\
\text { (attributed) }\end{array}$ & infrastructure & $\begin{array}{l}\text { ['S E. } \\
\text { Compagnies] } \\
\text { Slaaven Logie }\end{array}$ \\
\hline V 115-b & NA 4.TOPO 15.94 & Africa & Cape Colony & $\begin{array}{l}\text { 2nd of April } \\
1790\end{array}$ & $\begin{array}{l}\text { H.P. Wijting } \\
\text { Schull }\end{array}$ & infrastructure & $\begin{array}{l}\text { [on plan:] } \\
\text { Slaven vertrek }\end{array}$ \\
\hline V 132-a & NA 4.TOPO 15.83 & Africa & Cape Colony & c. 1790 & Anonymous & infrastructure & Slaavenwooning \\
\hline V 137-a & $\begin{array}{l}\text { Iziko William } \\
\text { Fehr Collection, E } \\
41\end{array}$ & Africa & Cape Colony & c. 1800 & I.N. Wildt & infrastructure & $\begin{array}{l}\text { Plan of the } \\
\text { Slave Lodje at } \\
\text { the Cape of } \\
\text { Good Hope }\end{array}$ \\
\hline V 137-b & NA 4.TOPO 15.92 & Africa & Cape Colony & c. 1788 & $\begin{array}{l}\text { Louis Michel } \\
\text { Thibault }\end{array}$ & infrastructure & $\begin{array}{l}\text { [on plan:] } \\
\text { Logement des } \\
\text { Eslaves }\end{array}$ \\
\hline $\begin{array}{l}\text { V } 138- \\
139\end{array}$ & NA 4.VEL 838 & Africa & Cape Colony & 1767 & C.F. Brink & infrastructure & $\begin{array}{l}\text { ['S E. } \\
\text { Compagnies] } \\
\text { Slaven Logie }\end{array}$ \\
\hline V 143 & NA 4.VEL 842 & Africa & Cape Colony & 1791 & J.W. Wernich & infrastructure & Slave prison \\
\hline V 144 & NA 4.TOPO 15.84 & Africa & Cape Colony & 1785 & Anonymous & infrastructure & $\begin{array}{l}\text { [on map:] SE } \\
\text { Comp. Slave } \\
\text { logie }\end{array}$ \\
\hline V 145 & NA 4.TOPO 15.85 & Africa & Cape Colony & $1785-1790$ & Anonymous & infrastructure & $\begin{array}{l}\text { [on map:] De } \\
\text { Slaven Logie }\end{array}$ \\
\hline V 152-a & $\begin{array}{l}\text { Atlas van Stolk, } \\
\text { TOP } 1055\end{array}$ & Africa & Cape Colony & 1764 & Johannes Rach & depiction & $\begin{array}{l}\text { [depiction of } \\
\text { enslaved people, } \\
\text { working] }\end{array}$ \\
\hline V 152-b & PNRI, BW 72 & Africa & Cape Colony & $1770-1780$ & $\begin{array}{l}\text { Johannes Rach, } \\
\text { apprentice or } \\
\text { follower }\end{array}$ & depiction & $\begin{array}{l}\text { [depiction of } \\
\text { enslaved people, } \\
\text { working] }\end{array}$ \\
\hline
\end{tabular}




\begin{tabular}{|c|c|c|c|c|c|c|c|}
\hline $\begin{array}{l}\text { V } 282- \\
283-b\end{array}$ & Private Collection & Africa & False Bay & 1787 & Jan Brandes & depiction & $\begin{array}{l}\text { [depiction of } \\
\text { enslaved people, } \\
\text { working] }\end{array}$ \\
\hline V 340-b & NA 4.TOPO 15.95 & Africa & Plettenberg Bay & 1789 & Anonymous & infrastructure & $\begin{array}{l}\text { [on plan:] Slaaf } \\
\text { Vertrek }\end{array}$ \\
\hline VI 108 & NA 4.VEL 869 & India & Surat & 1698 & Anonymous & infrastructure & $\begin{array}{l}\text { T slaven secreet; } \\
\text { 'T Slaven-huijs }\end{array}$ \\
\hline VI $113-c$ & $\begin{array}{l}\text { Book publication } \\
\text { Begin ende } \\
\text { Voortgangh }\end{array}$ & India & Surat & c. 1634 & Anonymous & depiction & $\begin{array}{l}\text { [depiction of } \\
\text { enslaved people, } \\
\text { working] }\end{array}$ \\
\hline VI 165 & NA 4.MIKO W37 & India & $\begin{array}{l}\text { Coasts of Malabar and } \\
\text { Coromandel }\end{array}$ & $1752-1757$ & Pieter de Bevere & allegorical & $\begin{array}{l}\text { [depiction of } \\
\text { enslaved people } \\
\text { in the cartouche, } \\
\text { allegorical] }\end{array}$ \\
\hline VI 239 & NA 4.VEL 619.48 & India & Cochin & $1690-1700$ & Anonymous & infrastructure & $\begin{array}{l}\text { Slaven quartier } \\
\text { boven de } \\
\text { matroses logis }\end{array}$ \\
\hline VI 361 & NA 4.VEL 1090 & India & Pulicat & 1708 & $\begin{array}{l}\text { Jan Christiaensz } \\
\text { Toorzee } \\
\text { (attributed) }\end{array}$ & infrastructure & Slaven kamertje \\
\hline VI 381 & $\begin{array}{l}\text { UBL, Bodel } \\
\text { Nijenhuis 002-09- } \\
034\end{array}$ & India & Bimilipatnam & 1756 & C.P. Keller & infrastructure & slave vertrekjes \\
\hline VI 382-a & $\begin{array}{l}\text { UBL, Bodel } \\
\text { Nijenhuis 002-09- } \\
040\end{array}$ & India & Bimilipatnam & 1756 & C.P. Keller & infrastructure & $\begin{array}{l}\text { [on plan:] Slaaf } \\
\text { kamer }\end{array}$ \\
\hline VI 402 & $\begin{array}{l}\text { Rijksmuseum, SK- } \\
\text { A-4282 }\end{array}$ & India & Hooghly & 1665 & $\begin{array}{l}\text { H. van } \\
\text { Schuylenburgh }\end{array}$ & depiction & $\begin{array}{l}\text { [depiction of } \\
\text { enslaved people, } \\
\text { working] }\end{array}$ \\
\hline VI 404-b & Private Collection & India & Chinsura & c. 1817 & Anonymous & depiction & $\begin{array}{l}\text { [depiction of } \\
\text { enslaved people, } \\
\text { working] }\end{array}$ \\
\hline VI 405 & $\begin{array}{l}\text { Rijksmuseum, SK- } \\
\text { A-4283 }\end{array}$ & India & Cossimbazar & c. 1665 & $\begin{array}{l}\text { H. van } \\
\text { Schuylenburgh }\end{array}$ & depiction & $\begin{array}{l}\text { [depiction of } \\
\text { enslaved people, } \\
\text { working] }\end{array}$ \\
\hline VI 410 & NA 4.VEL 1106 & India & Patna & c. 1733 & Anonymous & infrastructure & $\begin{array}{l}\text { Plaats daar } \\
\text { eenige slaave } \\
\text { huysjes staan }\end{array}$ \\
\hline VII 329-a & $\begin{array}{l}\text { NA 1.04.02 7577, } \\
\text { fols. } 92-93\end{array}$ & Indonesia & Batavia (Kuiper island) & 1769 & Johannes Rach & depiction & $\begin{array}{l}\text { [depiction of } \\
\text { enslaved people, } \\
\text { working] }\end{array}$ \\
\hline VII 332 & Private Collection & Indonesia & Batavia & 1779 & Jan Brandes & depiction & $\begin{array}{l}\text { [depiction of } \\
\text { enslaved people, } \\
\text { working] }\end{array}$ \\
\hline VII 363 & $\begin{array}{l}\text { UBL, Bodel } \\
\text { Nijenhuis 002-10- } \\
024\end{array}$ & Indonesia & Fort Nassau (Sulawesi) & c. 1775 & J.A. Mol & infrastructure & $\begin{array}{l}\text { Slaven } \\
\text { woningen }\end{array}$ \\
\hline
\end{tabular}

Table 1. Database slavery on manuscript VOC maps, plans and views 